\title{
Le mutazioni alla base della malattia di Fabry: come orientarsi tra quelle che causano e quelle che non causano la malattia
}

\section{Giovanni Duro}

\begin{abstract}
Mutations underlying Fabry's disease: how to get oriented between those that cause and those that do not cause the disease

Anderson-Fabry disease is a rare, progressive, multisystem, accumulation's disorder caused by partial or total deficiency of the lysosomal enzyme $\alpha$-GAL A. Fabry disease is an X-linked lysosomal enzymopathy determined by mutations in the GLA gene, coding for $\alpha$-GAL A. To date, almost 900 mutations in Fabry patients have been described.

Results: In our laboratories we studied the genetic and enzymatic alterations on almost 17,000 patients with systemic symptoms referable to Fabry disease. In $46 \mathrm{I}$ of these patients, exonic mutations in GLA were so classified: $73.5 \%$ of the mutations have been associated with classical phenotype, $17.2 \%$ with atypical variants, and $9.4 \%$ with GVUS.

Conclusions: The considerable number of patients we studied, allowed us to demonstrate that there is a complete relationship between symptoms, genetic and enzymatic findings and Lyso-GB3 accumulation. Our study shows that in all patients with causative mutation, who have absent or reduced enzymatic activity, we find hematic Lyso-GB3 accumulation, commensurate with the type of mutation and always according with the patient symptomatology.
\end{abstract}
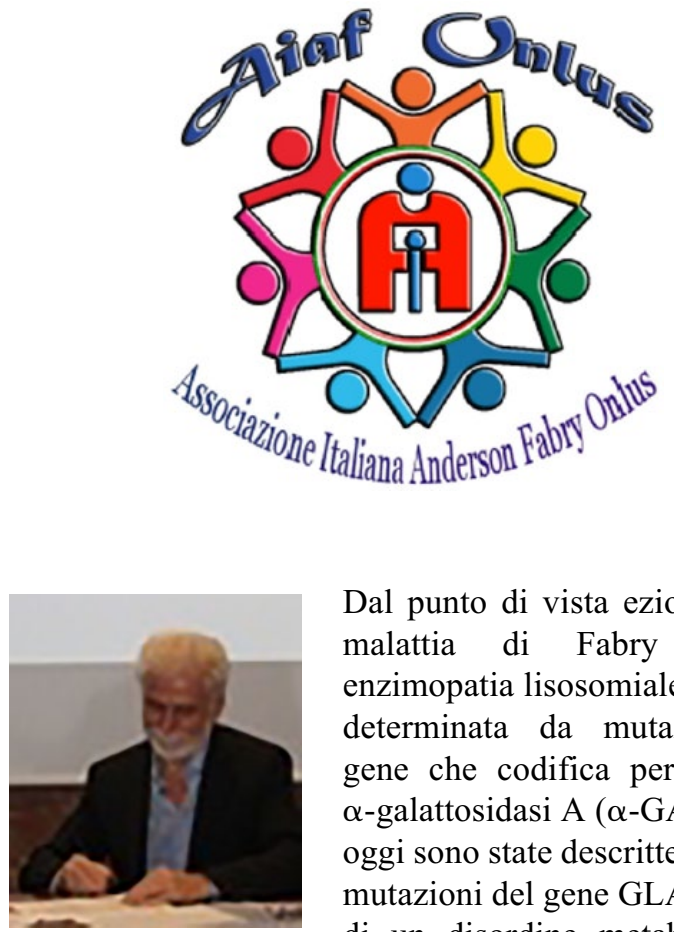

Dal punto di vista eziologico, la malattia di Fabry è una enzimopatia lisosomiale X-linked determinata da mutazioni nel gene che codifica per l'enzima $\alpha$-galattosidasi A ( $\alpha$-GAL A). Ad oggi sono state descritte circa 900 mutazioni del gene GLA. Si tratta di un disordine metabolico, da accumulo lisosomiale, caratterizzato dal deficit funzionale dell'enzima $\alpha$-GAL A. Tale deficit determina un'alterazione del metabolismo di alcuni glicosfingolipidi, prevalentemente globotriaosilceramide (Gb3), che di conseguenza si accumula nei lisosomi di numerosi tipi cellulari, soprattutto nelle cellule dell'endotelio vascolare. Ciò provoca manifestazioni cliniche sistemiche e complicanze di natura renale, cardiaca e cerebrovascolare o una combinazione di esse, che possono portare, intorno alla quinta decade di vita, ad una morte prematura. Oltre al fenotipo classico, sono identificati un numero sempre crescente di individui con un fenotipo attenuato, varianti atipiche. In letteratura sono riportati numerosi casi di malattia di Fabry, ad esordio tardivo e sintomatologia

IBIM-CNR-Palermo. Responsabile Centro di Ricerca e Diagnosi malattie da accumulo lisosomiale

\section{Corresponding author:}

Professor Giovanni Duro, Direttore di Ricerca IBIM-CNR Palermo, Docente di Genetica delle Malattie da Accumulo. Scuola di Medicina UniPA, IBIM-CNR, Via Ugo La Malfa I53, 90146 Palermo, Italy.

E-mail: duro@ibim.cnr.it http://www.ibim.cnr.it/index.php/giovanni-duro 
sfumata, che manifestano sintomi a carico di un solo organo. A causa della natura X-linked della malattia di Fabry, le femmine sono state a lungo considerate solo portatrici, mentre in realtà possono sviluppare notevoli segni e sintomi correlati alla malattia di Fabry, anche se il fenotipo è generalmente meno grave. Le pazienti con malattia di Fabry mostrano la malattia in modo "variabile", a causa di differenze nella lyonizzazione del cromosoma $X$. La diagnosi di malattia di Fabry risulta ancora oggi difficile proprio per le peculiarità della malattia, che si presenta con manifestazioni cliniche sovrapponibili a quelle di altre patologie, ed un'ampia possibilità di diagnosi differenziali che coinvolgono diverse specializzazioni mediche. L'errore diagnostico è un rischio concreto che determina una sottostima del reale numero di soggetti affetti. Il sospetto della malattia viene avanzato in base ai dati clinici e a quelli anamnesticofamiliari, e viene infine confermato attraverso analisi genetiche e biochimiche quali l'individuazione dell'alterazione genica specifica e il dosaggio dell'attività dell' $\alpha$-GAL A, che può essere nulla o deficitaria. Anche la determinazione dei substrati dell'enzima $\mathrm{Gb3}$, e la sua forma deacilata Lyso-Gb3, fornisce un supporto diagnostico.

Nei nostri laboratori abbiamo studiato le alterazioni genetiche ed enzimatiche in circa 17,000 pazienti con sintomi sistemici riconducibili alla malattia di Fabry. In 461 di questi pazienti sono state riscontate mutazioni esoniche in GLA così distribuite: il $73.5 \%$ delle mutazioni erano associate al fenotipo classico, il $17.2 \%$ alle varianti atipiche, e il $9.4 \%$ erano varianti genetiche il cui significato rimane ad oggi sconosciuto (GVUS).

Il numero significativo di pazienti studiati ci ha consentito di dimostrare che esiste una completa corrispondenza tra sintomi, genetica, enzimatica e accumulo di Lyso-GB3 e/o GB3.

Il nostro studio dimostra che in tutti i pazienti maschi e con mutazione causativa, in assenza o ridotta attività enzimatica, si riscontra sempre accumulo di Lyso-GB3 ematico, commisurato al tipo di mutazione e sempre in accordo con la sintomatologia del paziente.

La sintomatologia risulta essere classificabile come segue: severa, in assenza di attività enzimatica e in presenza di accumulo significativo, definita forma classica; lieve o organo-specifica, in presenza di attività enzimatica residua e in presenza di modesto accumulo, definite varianti atipiche; sfumata e/o assente, in presenza di attività nella norma e assenza di accumulo, mutazioni GVUS. Negli ultimi anni c'è stata molta confusione nel campo: molte mutazioni non causative sono state associate alla malattia di Fabry, e in qualche caso i pazienti sono stati sottoposti a terapia anche in presenza di un'attività enzimatica nella norma e assenza di accumulo. L'identificazione dei pazienti affetti da malattia di Fabry è possibile associando clinica, genetica, enzimatica e accumulo ematico di Lyso-GB3. Per quella che è la nostra esperienza il lyso-GB 3 ematico può essere considerato un marcatore attendibile molto utile. 